\title{
Boomerang Attacks on BLAKE-32
}

\author{
Alex Biryukov, Ivica Nikolić , and Arnab Roy \\ University of Luxembourg \\ $\{$ alex.biryukov, ivica.nikolic, arnab.roy\}@uni.lu
}

\begin{abstract}
We present high probability differential trails on 2 and 3 rounds of BLAKE-32. Using the trails we are able to launch boomerang attacks on up to 8 round-reduced keyed permutation of BLAKE-32. Also, we show that boomerangs can be used as distinguishers for hash/ compression functions and present such distinguishers for the compression function of BLAKE-32 reduced to 7 rounds. Since our distinguishers on up to 6 round-reduced keyed permutation of BLAKE-32 are practical (complexity of only $2^{12}$ encryptions), we are able to find boomerang quartets on a PC.
\end{abstract}

Keywords: SHA-3 competition, hash function, BLAKE, boomerang attack, cryptanalysis.

\section{Introduction}

The SHA-3 competition [6] will soon enter the third and final phase, by selecting 5 out of 14 second round candidates. The hash function BLAKE [2] is among these 14 candidates, and it is one of the few functions that has not been tweaked from the initial submission in 2008. Being an addition-rotation-xor (ARX) design, BLAKE is one of the fastest functions on various platforms in software. Indeed, among the fastest candidates, BLAKE has the highest published security level, i.e. the best published attacks work only on a small fraction of the total number of rounds. Few attacks, however, were published on the roundreduced compression function and keyed permutation of BLAKE-32 (which has 10 rounds). In [3] Ji and Liangyu present collision and preimage attacks on 2.5 rounds of the compression function of BLAKE-32. Su et al. 7] give near collisions on 4 rounds with a complexity of $2^{21}$ compression function calls. However, one can argue that the message modification they use, requires an additional effort of $2^{64}$ (see Sec. 51). Aumasson et al. in [1, among other, present near collisions on 4 rounds of the compression function with $2^{56}$ complexity, and impossible differentials on 5 rounds of the keyed permutation.

Our Contribution. We show various boomerang distinguishers on roundreduced BLAKE-32. Our analysis is based on the fact that BLAKE-32, being a keyed permutation, has some high probability differential trails on two

\footnotetext{
* This author is supported by the Fonds National de la Recherche Luxembourg grant TR-PHD-BFR07-031.
} 
and three rounds $\left(2^{-1}\right.$ on two and $2^{-7}$ on three rounds). Moreover, we can extend the three round trail to four rounds. First, we use these trails to build boomerang distinguishers for the round-reduced keyed permutation of BLAKE32 on up to 8 rounds. Then we extend the concept of boomerang distinguishers to hash functions. As far as we know, this is the first application of the standard boomerangs to hash function. An amplified boomerang attack applied to hash functions was presented in [4], however it was used in addition to a collision attack. Our boomerang attacks, on the other hand, are standalone distinguishers, and work in the same way as for block ciphers - by producing the quartet of plaintexts and ciphertexts (input chaining values and output chaining values). We also show how to obtain simpler zero-sum distinguisher from the boomerang and present such distinguishers for 4, 5, 6 rounds of BLAKE-32. Our final result is a boomerang distinguisher for 7 rounds of the compression function of BLAKE-32. The summary of our results is given in Table 1 .

Although in this paper we focus on BLAKE-32, our attacks can be easily extended to the other versions of BLAKE (with similar complexities and number of attacked rounds). The attacks do not contradict any security claims of BLAKE.

Table 1. Summary of the attacks on the compression function (CF) and the keyed permutation (KP) of BLAKE-32

\begin{tabular}{|c|c|c|c|c|}
\hline Attack & $\mathrm{CF} / \mathrm{KP}$ & Rounds & $\mathrm{CF} / \mathrm{KP}$ calls & Reference \\
\hline Free-start collisions & $\overline{\mathrm{CF}}$ & 2.5 & $2^{112}$ & 3 \\
\hline Near collisions $^{\text {a }}$ & $\overline{\mathrm{CF}}$ & 4 & $2^{21}$ & [7] \\
\hline Near collisions & $\mathrm{CF}$ & 4 & $2^{56}$ & 1 \\
\hline Impossible diffs. & $\mathrm{KP}$ & 5 & - & 1 \\
\hline Boomerang dist. & $\overline{\mathrm{CF}}$ & $\overline{4}$ & $2^{677}$ & Sec. 5] \\
\hline Boomerang dist. & $\mathrm{CF}$ & 5 & $2^{71.2}$ & Sec. 5 \\
\hline Boomerang dist. & $\mathrm{CF}$ & 6 & $2^{102}$ & Sec. 5 \\
\hline Boomerang dist. & $\mathrm{CF}$ & 6.5 & $2^{184}$ & Sec. [5] \\
\hline Boomerang dist. & $\mathrm{CF}$ & 7 & $2^{232}$ & Sec. 5 \\
\hline Boomerang dist. & $\mathrm{KP}$ & 4 & $2^{3}$ & Sec. [6] \\
\hline Boomerang dist. & $\mathrm{KP}$ & 5 & $2^{7.2}$ & Sec. 6 \\
\hline Boomerang dist. & $\mathrm{KP}$ & 6 & $2^{11.75}$ & Sec. [6] \\
\hline Boomerang dist. & $\mathrm{KP}$ & 7 & $2^{122}$ & Sec. [6] \\
\hline Boomerang dist. & $\mathrm{KP}$ & 8 & $2^{242}$ & Sec. 6 \\
\hline
\end{tabular}

${ }^{a}$ The attack assumes that message modification can be used anywhere in the trail.

\section{Description of BLAKE32}

The compression function of BLAKE-32 processes a state of 16 32-bit words represented as $4 \times 4$ matrix. Each word in BLAKE-32 has 32 bits. In the Initialization procedure, the state is loaded with a chaining value $h_{0}, \ldots, h_{7}$, a salt $s_{0}, \ldots, s_{3}$, constants $c_{0}, \ldots, c_{7}$, a counter $t_{0}, t_{1}$ as follows: 


$$
\left(\begin{array}{cccc}
v_{0} & v_{1} & v_{2} & v_{3} \\
v_{4} & v_{5} & v_{6} & v_{7} \\
v_{8} & v_{9} & v_{10} & v_{11} \\
v_{12} & v_{13} & v_{14} & v_{15}
\end{array}\right) \longleftarrow\left(\begin{array}{cccc}
h_{0} & h_{1} & h_{2} & h_{3} \\
h_{4} & h_{5} & h_{6} & h_{7} \\
s_{0} \oplus c_{0} & s_{1} \oplus c_{1} & s_{2} \oplus c_{2} & s_{3} \oplus c_{3} \\
t_{0} \oplus c_{4} & t_{0} \oplus c_{5} & t_{1} \oplus c_{6} & t_{1} \oplus c_{7}
\end{array}\right)
$$

After the Initialization, the compression function takes 16 message words $m_{0}, \ldots, m_{15}$ as inputs and iterates 10 rounds. Each round is composed of eight applications of $\mathrm{G}$ function. A column step:

$$
\mathrm{G}_{0}\left(v_{0}, v_{4}, v_{8}, v_{12}\right), \mathrm{G}_{1}\left(v_{1}, v_{5}, v_{9}, v_{13}\right), \mathrm{G}_{2}\left(v_{2}, v_{6}, v_{10}, v_{14}\right), \mathrm{G}_{3}\left(v_{3}, v_{7}, v_{11}, v_{15}\right)
$$

followed by the diagonal step:

$$
\mathrm{G}_{4}\left(v_{0}, v_{5}, v_{10}, v_{15}\right), \mathrm{G}_{5}\left(v_{1}, v_{6}, v_{11}, v_{12}\right), \mathrm{G}_{6}\left(v_{2}, v_{7}, v_{8}, v_{13}\right), \mathrm{G}_{7}\left(v_{3}, v_{4}, v_{9}, v_{14}\right)
$$

where $\mathbf{G}_{i}(i \in\{0, \ldots, 7\})$ depend on their indices, message words $m_{0}, \ldots, m_{15}$, constants $c_{0}, \ldots, c_{15}$ and round index $r$. At round $r, \mathrm{G}_{i}(a, b, c, d)$ is described with following steps:

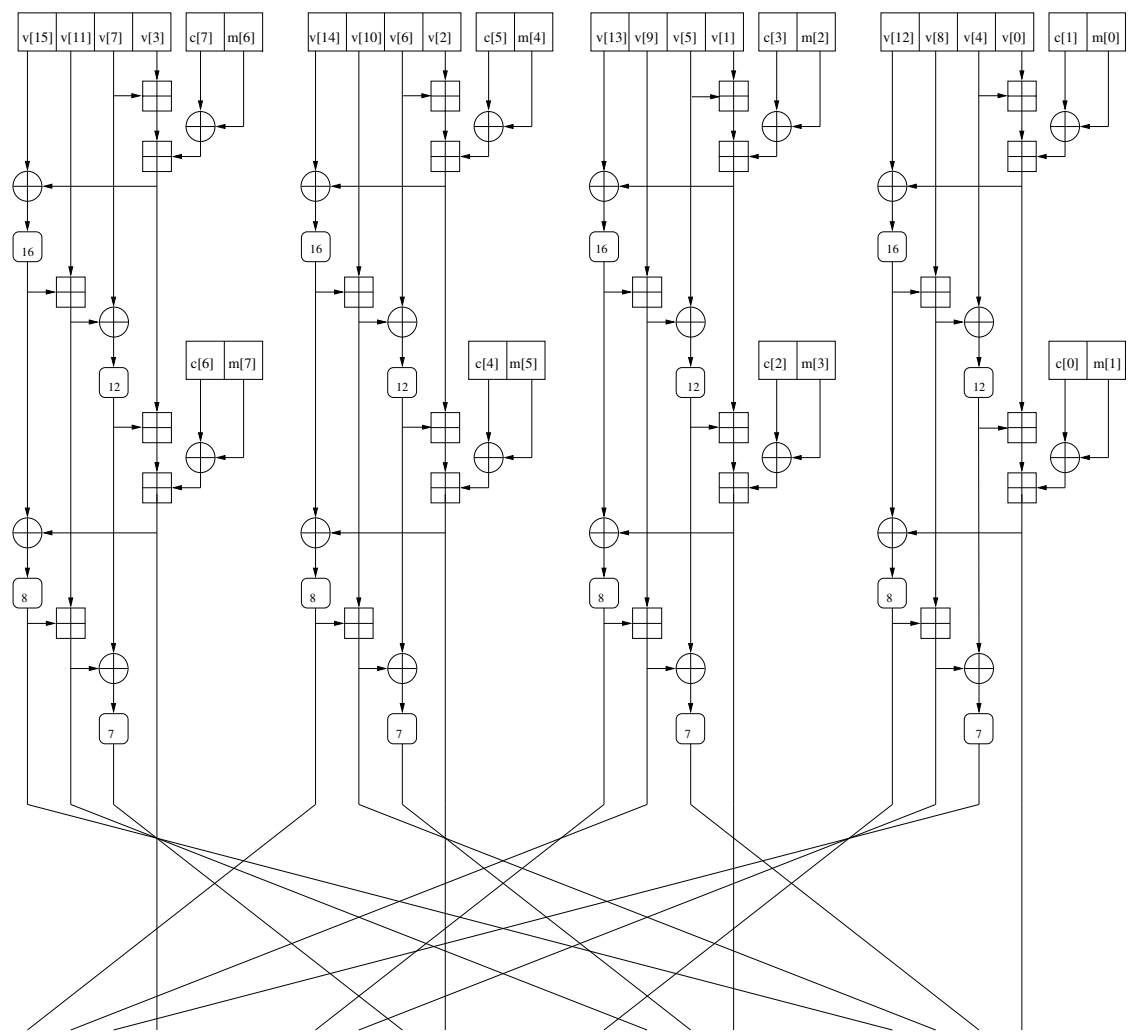

Fig. 1. Column step of round-0 


$$
\begin{aligned}
& 1: a \leftarrow a+b+\left(m_{\sigma_{r}(2 i)} \oplus c_{\sigma_{r}(2 i+1)}\right) \\
& 2: d \leftarrow(d \oplus a) \ggg 16 \\
& 3: c \leftarrow c+d \\
& 4: b \leftarrow(b \oplus c) \ggg 12 \\
& 5: a \leftarrow a+b+\left(m_{\sigma_{r}(2 i+1)} \oplus c_{\sigma_{r}(2 i)}\right) \\
& 6: d \leftarrow(d \oplus a) \ggg 8 \\
& 7: c \leftarrow c+d \\
& 8: b \leftarrow(b \oplus c) \ggg 7
\end{aligned}
$$

where $\sigma_{r}$ belongs to the set of permutations as specified in [2]. The Finalization procedure in BLAKE-32 is depicted as:

$$
\begin{aligned}
& h_{0}^{\prime} \leftarrow h_{0} \oplus s_{0} \oplus v_{0} \oplus v_{8} \\
& h_{1}^{\prime} \leftarrow h_{1} \oplus s_{1} \oplus v_{1} \oplus v_{9} \\
& h_{2}^{\prime} \leftarrow h_{2} \oplus s_{2} \oplus v_{2} \oplus v_{10} \\
& h_{3}^{\prime} \leftarrow h_{3} \oplus s_{3} \oplus v_{3} \oplus v_{11} \\
& h_{4}^{\prime} \leftarrow h_{4} \oplus s_{0} \oplus v_{4} \oplus v_{12} \\
& h_{5}^{\prime} \leftarrow h_{5} \oplus s_{1} \oplus v_{5} \oplus v_{13} \\
& h_{6}^{\prime} \leftarrow h_{6} \oplus s_{2} \oplus v_{6} \oplus v_{14} \\
& h_{7}^{\prime} \leftarrow h_{7} \oplus s_{3} \oplus v_{7} \oplus v_{15}
\end{aligned}
$$

where $h_{0}, \ldots, h_{7}$ is the initial chaining value and $v_{0}, \ldots, v_{15}$ is the state value after the ten rounds, and $h_{0}^{\prime}, \ldots, h_{7}^{\prime}$ are the words of the new chaining value.

\section{Boomerang Attacks on Block Ciphers and Compression Functions}

The boomerang attack 8 is a differential-type attack that exploits high probability differential trails in each half of a cipher $E$. When successful, it outputs a quartet of plaintexts and corresponding ciphertexts with some fixed particular differences between some of the pairs. This property can be used to distinguish the cipher from a random permutation, and in some cases, to recover the key.

Let us decompose the initial cipher $E$ into two ciphers $E_{0}, E_{1}$, i.e. $E=E_{1} \circ E_{0}$. Let $\Delta \rightarrow \Delta^{*}$ be some differential trail for $E_{0}$ that holds with probability $p$ and $\nabla \rightarrow \nabla^{*}$ be a trail for $E_{1}$ with probability $q$. We start with a pair of plaintexts $\left(P_{1}, P_{2}\right)=\left(P_{1}, P_{1} \oplus \Delta\right)$ and produce a pair of corresponding ciphertexts $\left(C_{1}, C_{2}\right)=\left(E\left(P_{1}\right), E\left(P_{2}\right)\right)$. Then we produce a new pair of ciphertext $\left(C_{3}, C_{4}\right)=\left(C_{1} \oplus \nabla^{*}, C_{2} \oplus \nabla^{*}\right)$, decrypt this pair, and get the corresponding pair of plaintexts $\left(P_{3}, P_{4}\right)=\left(E^{-1}\left(C_{3}\right), E^{-1}\left(C_{4}\right)\right)$. The difference $P_{3} \oplus P_{4}$ is $\Delta$ with probability at least $\left.p^{2} q^{2}: 1\right)$ the difference $E_{0}\left(P_{1}\right) \oplus E_{0}\left(P_{2}\right)$ is $\Delta^{*}$ with probability $p ; 2)$ the differences $E_{1}^{-1}\left(C_{1}\right) \oplus E_{1}^{-1}\left(C_{3}\right), E_{1}^{-1}\left(C_{2}\right) \oplus E_{1}^{-1}\left(C_{4}\right)$ are both $\nabla$ with probability $\left.q^{2} ; 3\right)$ when 1$), 2$ ) hold, then the difference $E_{1}^{-1}\left(C_{3}\right) \oplus E_{1}^{-1}\left(C_{4}\right)$ is $\Delta^{*}$ (with probability $p q^{2}$ ) and $E^{-1}\left(C_{3}\right) \oplus E^{-1}\left(C_{4}\right)$ is $\Delta$ with probability $p^{2} q^{2}$.

We would like to address a couple of issues. First, the boomerang distinguisher can be used even in the case when it returns a pair $\left(P_{3}, P_{4}\right)$ with a difference 
$P_{3} \oplus P_{4}$ specified only in certain bits (instead of the full plaintext). When the difference is specified in $t$ bits $(t<n)$, then the probability of the boomerang (in order to be used as a distinguisher) should be higher than $2^{-t}$, i.e. $p^{2} q^{2}>2^{-t}$. Second, the real probability of the boomerang is $\hat{p}^{2} \hat{q}^{2}$, where $\hat{p}, \hat{q}$ are so-called amplified probabilities, defined as:

$$
\hat{p}=\sqrt{\sum_{\Delta^{*}} P\left[\Delta \rightarrow \Delta^{*}\right]^{2}}, \hat{q}=\sqrt{\sum_{\nabla} P\left[\nabla \rightarrow \nabla^{*}\right]^{2}}
$$

Since finding these values is hard, in some cases, we try to get experimental results for the probability of the boomerang. We run a computer simulation, start the boomerang with a number of pairs with some prefixed difference $\Delta$, and count the number of returned pairs that have the same difference $\Delta$. Obviously the ratio of the returned pairs to the launched pairs is the probability of the boomerang.

The main obstacle for applying the boomerang attack to compression functions, is that in general, the compression functions are non-invertible. Hence, after obtaining the pairs $\left(C_{3}, C_{4}\right)$ from $\left(C_{1}, C_{2}\right)$, one cannot go backwards and obtain the pair $\left(P_{3}, P_{4}\right)$. One way to deal with this is to switch to amplified boomerang attacks 5. However, this type of boomerangs usually has lower probability, and more importantly, since it requires internal collisions, in the case when the underlying compression functions are double pipes, the attack complexity becomes higher than in a trivial attack.

Indeed, the standard boomerang attack can be used as a differential distinguisher for a compression function $F$. The idea is to start the attack in the middle of $F$ and then go forward and backwards to obtain the quartets, thus escaping the feedforward. Let $F(H)$ be obtained from some invertible function $f(H)$ with a feedforward, for example Davies-Meyer mode $F(H)=f(H) \oplus H$. As in the attack on block ciphers, first step is to decompose $f$ into two functions $f_{0}, f_{1}$ and to find two differential trails for $f_{0}$ and $f_{1}$ (further we use the same notation as in the attacks on block ciphers). We start with four states $S_{1}, S_{2}, S_{3}, S_{4}$ at the end of the function $f_{0}$ (beginning of $f_{1}$ ) such that $S_{1} \oplus S_{2}=S_{3} \oplus S_{4}=\Delta^{*}$ and $S_{1} \oplus S_{3}=S_{2} \oplus S_{4}=\nabla$. From these states we obtain the initial states (input chaining values) $P_{i}$ and the final states (output chaining values without the feedforward) $C_{i}$, i.e. $P_{i}=f_{0}^{-1}\left(S_{i}\right), C_{i}=f_{1}\left(S_{i}\right), i=1, \ldots, 4$. Then with probability at least $p^{2} q^{2}$ we have:

$$
\begin{array}{rrr}
P_{1} \oplus P_{2}=\Delta, & P_{3} \oplus P_{4}=\Delta \\
C_{1} \oplus C_{3}=\nabla^{*}, & C_{2} \oplus C_{4}=\nabla^{*} .
\end{array}
$$

Extending the following attack to the whole compression function $F$ is trivial - we just have to take into account that $C_{i}=f\left(P_{i}\right)=F\left(P_{i}\right) \oplus P_{i}$. For the boomerang quartet $\left(P_{1}, P_{2}, P_{3}, P_{4}\right)$ we get:

$$
\begin{array}{lr}
P_{1} \oplus P_{2}=\Delta, & P_{3} \oplus P_{4}=\Delta \\
{\left[F\left(P_{1}\right) \oplus P_{1}\right] \oplus\left[F\left(P_{3}\right) \oplus P_{3}\right]=\nabla^{*},} & {\left[F\left(P_{2}\right) \oplus P_{2}\right] \oplus\left[F\left(P_{4}\right) \oplus P_{4}\right]=\nabla^{*}}
\end{array}
$$


For a random $n$-bit compression function $F$, the complexity of finding the quartet $\left(P_{1}, P_{2}, P_{3}, P_{4}\right)$ with the above relations (2),(3), is around $2^{n}$. Hence when $p^{2} q^{2}>2^{-n}$ one can launch a boomerang attack and thus obtain a distinguisher for $F$. The distinguisher becomes even more powerful if the attacker finds several boomerang quartets with the same differences $\Delta, \nabla^{*}$.

A zero-sum distinguisher, can be obtained based on the boomerangs. If in (3), we XOR the two equations, we get:

$$
\begin{aligned}
0 & =\left[F\left(P_{1}\right) \oplus P_{1}\right] \oplus\left[F\left(P_{3}\right) \oplus P_{3}\right] \oplus \nabla^{*} \oplus\left[F\left(P_{2}\right) \oplus P_{2}\right] \oplus\left[F\left(P_{4}\right) \oplus P_{4}\right] \oplus \nabla^{*}= \\
& =F\left(P_{1}\right) \oplus F\left(P_{2}\right) \oplus F\left(P_{3}\right) \oplus F\left(P_{4}\right) \oplus\left(P_{1} \oplus P_{2}\right) \oplus\left(P_{3} \oplus P_{4}\right)= \\
& =F\left(P_{1}\right) \oplus F\left(P_{2}\right) \oplus F\left(P_{3}\right) \oplus F\left(P_{4}\right) \oplus \Delta \oplus \Delta= \\
& =F\left(P_{1}\right) \oplus F\left(P_{2}\right) \oplus F\left(P_{3}\right) \oplus F\left(P_{4}\right)
\end{aligned}
$$

Finding a zero-sum distinguisher for a random permutation requires $2^{n / 4}$ encryptions. However, since we have the additional conditions on the plaintexts (the XORs of the pairs are fixed), the complexity rises to $2^{n / 2}$.

It is important to notice that to produce the quartet (for the boomerang or the zero-sum boomerang) one has to start not necessarily from the middle states $\left(S_{1}, S_{2}, S_{3}, S_{4}\right)$. For example, one can start from two input chaining values $\left(P_{1}, P_{2}\right)=\left(P_{1}, P_{1} \oplus \Delta\right)$, produce the values $\left(S_{1}, S_{2}\right)=\left(f_{0}\left(P_{1}\right), f_{0}\left(P_{2}\right)\right)$, then obtain the values for the two other middle states $\left(S_{3}, S_{4}\right)=\left(S_{1} \oplus \nabla, S_{2} \oplus \nabla\right)$, and finally get the two input chaining values $\left(P_{3}, P_{4}\right)=\left(f_{0}^{-1}\left(S_{3}\right), f_{0}^{-1}\left(S_{4}\right)\right)$ and the four output chaining values $\left(f_{1}\left(S_{1}\right) \oplus P_{1}, f_{1}\left(S_{2}\right) \oplus P_{2}, f_{1}\left(S_{3}\right) \oplus P_{3}, f_{1}\left(S_{4}\right) \oplus P_{4}\right)$. Clearly, the probability of the boomerang stays the same. Starting from the beginning (or from some other particular state before the feedforward) can be beneficial in the cases when one wants to use message modification or wants to have some specific values in one of the four states (as shown further in the case of BLAKE-32).

\section{Round-Reduced Differential Trails in BLAKE-32}

In order to obtain good differential trails in BLAKE we exploit the structure of the message word permutation. In fact we can easily obtain good 2-round differential trail. The idea is to choose a message word $m_{j}$ such that

- It appears at Step 1(Case1) or at Step 5(Case2) in $\mathrm{G}_{i}(0 \leq i \leq 3)$ at round- $r$ and

- Also appears at Step 5 in $\mathrm{G}_{i}(4 \leq i \leq 7)$ at round- $(r+1)$.

If we choose the message word with the above mentioned strategy then with a suitable input difference we may pass 1.5 rounds for free (i.e. with probability 1 ).

\footnotetext{
${ }^{1}$ This holds only when the difference between the messages is fixed as well. Otherwise, the complexity is only $2^{n / 2}$.

${ }^{2}$ A similar technique was used in the analysis presented in [7].
} 
Observation 1. A 2-round differential trail can be obtained in BLAKE-32 with probability $2^{-1}$.

Proof. Choose two rounds with a message word $m_{j}$ as described previously. In

- Case1, we choose $\Delta m_{j}=\Delta a=0 \times 80000000$

- Case2, we choose $\Delta m_{j}=\Delta a=\Delta d=0 \times 80000000$

in the corresponding $\mathrm{G}$ function (see Fig. 2). After 1.5 rounds we get $\Delta v_{k}=$ $0, \forall k \in\{0, \ldots, 15\}$ with probability 1 . In the next half of the second round because of our choice of message word and suitable difference, we get one active bit only at step 7 in the corresponding G function (see Fig. 3). Hence we get a differential trail with probability $2^{-1}$.

Remark 1. In Case1 if $\Delta m_{j}$ and $\Delta a$ have any active bits other than MSB then at round- $r$, probability of the trail is $2^{-t}$ (where $t$ is the number of active bits in $\Delta m_{j}(=\Delta a)$ at round- $\left.r\right)$ and at round- $(r+1)$ the probability is $2^{-s}$, where $s=2 t-1,2 t, 2 t+1$ (depending on the position of active bits). So in this case the probability for two rounds will be $1 / 2^{s+t}$. Also if $m_{j}$ appears at Step 1 in $\mathrm{G}_{i}(4 \leq i \leq 7)$ at round- $(r+1)$ then probability of a 2 -round differential trail decreases further.

Remark 2. In Case1 if $\Delta m_{j}=\Delta a=\Delta$, such that $\Delta$ has two active bits at $i$ th and $(i+16)$ th position and $m_{j}$ appears at step 1 in $\mathrm{G}_{i}(4 \leq i \leq 7)$ at round- $(r+1)$ then we have 2-round differential trail with probability $2^{-8-1}\left(=2^{-9}\right)$ when $i$ th bit is the MSB and $\geq 2^{-12-2}\left(=2^{-14}\right)$ otherwise.

In order to construct 3-round trails from these 2-round differential trails we may simply add one more round at the beginning. The occurrence of the chosen message word in this one round does not affect much in terms of probability of the difference propagation.

Observation 2. A 3-round differential trail may be obtained from the above described two round differential trail with probability $2^{-s}$, where $s=6,7$ or 8

Proof. After obtaining 2-round differential trail with probability $2^{-1}$ (Case1), we add one more round(say, round- $(r-1))$ at the beginning. The probability of this one round differential trail may vary depending on the position of the message word $m_{j}$. Suppose the message word occurs in $\mathbf{G}_{l}$ (for some index $l$ ) at round $r$. Then at round $r-1$ :

- If the message word is in $\mathrm{G}_{i}(0 \leq i \leq 3)$ or at step 1 of $\mathrm{G}_{i}(4 \leq i \leq 7)$, probability of this one round trail is $2^{-6}$.

- If the message word occurs at step 5 of $\mathrm{G}_{l+4}$, we get differential trail with probability $2^{-5}$ for this one round.

For all other cases the probability of this one round differential trail is $2^{-7}$. Hence we get a 3 -round differential trail with probability $2^{-7}, 2^{-6}$ and $2^{-8}$ respectively. 

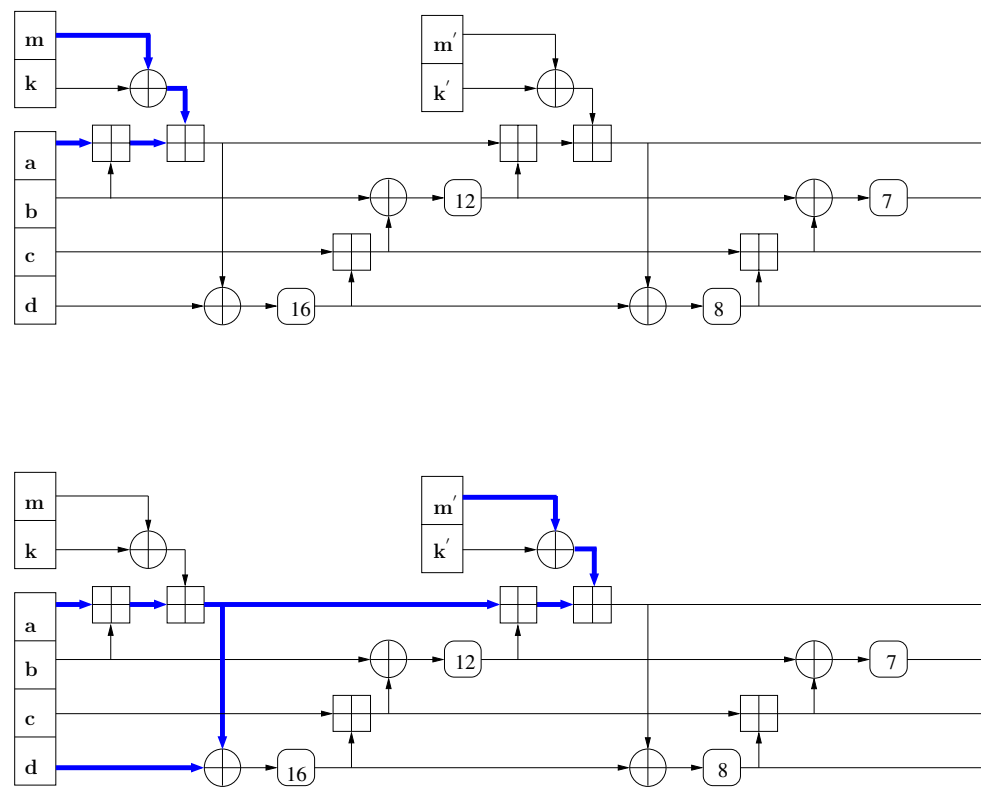

Fig. 2. Two possible differential trails for $\mathrm{G}$ at the beginning of 2-round trail. The top trail is Case1, while the bottom is Case2.
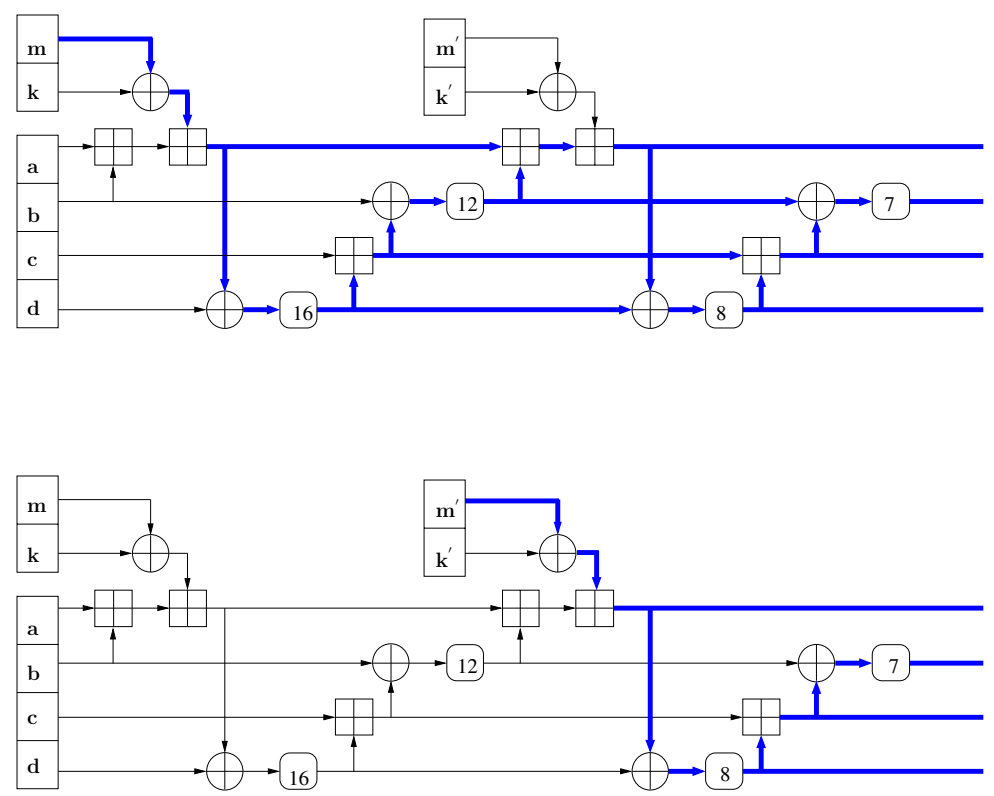

Fig. 3. Two possible differential trails for $\mathrm{G}$ at the end of 2-round trail. The top trail is when the message with the difference appears at Step 1, and the bottom at Step 5. 
Remark 3. This 3-round differential trail can be extended for half more round in the forward direction. If we add half round at the end of this three rounds and if the chosen message word does not occur there then we can get 3.5-round trail with probability $\geq 2^{-24-8}\left(=2^{-32}\right)$.

For this three round differential trail we have to inject two distinct input differences at $v_{12}$ and $v_{13}$ which correspond to the same counter $t_{0}$. In order to obtain a 3-round differential trail with consistent input differences at the states corresponding to the counters $t_{0}$ and $t_{1}$ we use a 2 -round trail with lower probability.

Observation 3. Let $\Delta a=\Delta c=\Delta$ such that $\Delta$ has only ith and $(i+16)$ th bits active. For a $G$ function if there is no difference in the message words then the differential trail $(\Delta, 0, \Delta, 0) \rightarrow(\Delta, 0,0,0)$ occurs with probability $2^{-3}$ if ith bit is the $M S B$ and with probability $2^{-6}$ otherwise.

Observation 4. A 3-round differential trail with input difference consistent with counters $\left(t_{0}, t_{1}\right)$ may be obtained with probability $2^{-21}$ or at least $2^{-36}$.

Proof. Starting with $\Delta m_{j}=\Delta a=\Delta=0 \times 80008000$ we obtain a 2-round differential trail with probability $2^{-9}$ (as described in Remark 2 ). Then we add one more round at the beginning. The position of the message word $m_{j}$ in this one round determines which three rounds we should consider in order to obtain the 3-round trail. Such three rounds may be found if we start with round-4. Now in this one round(added at the beginning) we have two G functions with differences as described in Observation 3 and one $\mathrm{G}$ function with difference $\left(\Delta_{1}, \Delta_{2}, \Delta, 0\right) \rightarrow(0,0, \Delta, 0)$ (with the message difference at step 5 in it). So probability for this one round is $2^{-6-6}=2^{-12}$. Hence we get a 3 -round trail with probability $2^{-21}$. If $\Delta$ has two active bits (e.g. 0x00080008) then probability of this one round at the beginning may be at least $2^{-12-10}=2^{-22}$ and probability of the 2 -round trail is at least $2^{-14}$. Hence we get 3 -round differential trail with probability at least $2^{-36}$.

The choice of message word for the 3-round differential trail specified in Observation 4 is available if we start with round- 4 and the input differences for the states corresponding to the counters are $\Delta v_{12}=\Delta v_{13}=\Delta v_{14}=\Delta v_{15}=0$.

A similar 2-round and 3-round differential trails exist for BLAKE-64.

\section{Boomerang Attacks on the Compression Function of BLAKE-32}

The high probability round-reduced differential trails in the permutation of BLAKE-32 can be used to attack the compression function and find boomerang distinguishers. However, due to the Initialization procedure, there are a few requirements on the trails. First, since the block index is copied twice, the initial differences in $v_{12}$ and $v_{13}$, as well as the differences in $v_{14}$ and $v_{15}$, have to be the same. Second, even in the case when the attacker has a trail with initial 
differences consistent to the above requirement, if he uses message modification techniques in the higher rounds of the trail, he might end up with inconsistent initial states. For example, if the attacker uses some $k$-round trail and starts fixing the values of the state and the messages at round $k$, and then goes backward, he can obtain two states with some predefined difference (as the one predicted by the trail). However, the probability that these two states are consistent with the Initialization procedure is $2^{-64}$ (if $v_{12} \oplus v_{13}=c_{4} \oplus c_{5}$ and $v_{14} \oplus v_{15}=c_{6} \oplus c_{7}$ ). Note that if one of the states is consistent, then the other one is consistent as well (if the attacker used trails with appropriate initial difference). Therefore, using message modification techniques in later steps of the trail is not trivial (without increasing the complexity of the attack). On the other hand, the modification can still be used at the beginning because the attacker starts with two states consistent with the Initialization procedure.

For the boomerang attack on 4 rounds of the compression function of BLAKE32 we can use two trails each on 2 rounds (see Table 21). Since the probability of these trails is only $2^{-1}$, the probability of the boomerang is $2^{-4}$. To create a quartet of states, consistent with the Initialization procedure, we start with a pair of states $\left(P_{1}, P_{2}\right)$ that have a difference $\Delta$ (note that $\Delta$ does not have a difference in the "block index" words) and consistent with the Initialization words $v_{12}, v_{13}, v_{14}, v_{15}$ in both of the states, then go two rounds forward and obtain the pair $\left(S_{1}, S_{2}\right)$. Then we produce the pair $\left(S_{3}, S_{4}\right)=\left(S_{1} \oplus \nabla, S_{2} \oplus \nabla\right)$ and go backwards two rounds to get the pair of initial states $\left(P_{3}, P_{4}\right)$. The probability that $P_{3}$ (and therefore $P_{4}$ ) is consistent with the Initialization is $2^{-64}$. Also, from $S_{1}, S_{2}, S_{3}, S_{4}$ we go forward two rounds, produce the outputs and apply the Finalization to get the new chaining values. Note that Finalization is linear, hence the differential trail (with XOR difference) holds with probability 1. Therefore, we can produce the boomerang quartet with a complexity of $4 \cdot 2^{4+64}=$ $2^{70}$ calls to the 4-round reduced compression function of BLAKE-32.

The boomerang attack on 5 rounds is rather similar. We only need one of the trails to be on 3 rounds, instead of 2 (see Table 3). Such a trail has a probability of $2^{-7}$, and we use two round trail with $2^{-3}$, hence the boomerang has a probability of $2^{-2 \cdot 3-2 \cdot 7}=2^{-20}$ and the whole attack (taking into account the Initialization) has a complexity of around $4 \cdot 2^{20+64}=2^{86}$ compression function calls.

For the boomerang attack on 6 rounds we will use two 3-round trails (see Table 4). However, we cannot use the optimal trails (the ones that hold with around $2^{-7}$ ) because the starting difference in each such trail is inconsistent with the Initialization procedure. Therefore, for the top trail of the boomerang we will use a trail which has lower probability $2^{-34}$ but has no differences in any of the "block index" words $\left(v_{12}, v_{13}, v_{14}, v_{15}\right)$. For the bottom trail we can use an optimal trail. The complexity of this boomerang distinguisher on 6 rounds becomes $4 \cdot 2^{2 \cdot 34+2 \cdot 7+64}=2^{148}$ calls.

Note, for the top trails for 5 and 6 round boomerangs (see Table 344), we did not use the best trails with probability $2^{-1}, 2^{-21}$, but instead used trails with lower probability $\left(2^{-3}, 2^{-34}\right)$. We found that if we use the best trails, then the boomerang does not work, most likely because of the slow diffusion. We cannot 
get four states in the middle (after the third round), that have pairwise $\Delta^{*}$ and $\nabla$ difference ( $\Delta^{*}$ is the end difference of the top trail). However, if we take other trails, as the ones we have taken, the boomerang quartet can be obtained - we confirmed this experimentally, by producing a boomerang quartet.

Each of the above attacks can be improved if we take into account the amplified probabilities for the boomerang attack and if we use message modification. We can obtain the amplified probabilities (and the total probabilities) of the boomerang experimentally: we start with a number of plaintext pairs with the required difference $\Delta$, and then check how many of the returned (by the boomerang) differences are $\Delta$. Also, in the first round, for one side of the boomerang we use message modification, i.e. we pass this round with probability 1. Using these two approaches, we got the following results: the boomerang on 4 rounds has a probability $2^{-1}$, on 5 rounds $2^{-5.2}$, and on 6 rounds $2^{-36}$. Hence, the attack complexity for 4 rounds drops to $4 \cdot 2^{1+64}=2^{67}$, for 5 rounds to $4 \cdot 2^{5.2+64}=2^{71.2}$, and for 6 rounds to $4 \cdot 2^{36+64}=2^{102}$ compression function calls. An example of boomerang quartet for 6 rounds, with the first pair of plaintext consistent to the Initialization, while only the difference in the second is consistent, and therefore obtained with around $4 \cdot 2^{36}$ compression function calls, is given in Table 9. The complexities of the boomerang distinguishers for 4,5, and 6 round are bellow $2^{128}$, therefore they can be used as zero-sum boomerang distinguishers, i.e. $P_{1} \oplus P_{2}=P_{3} \oplus P_{4}=\Delta$ and $F\left(P_{1}\right) \oplus F\left(P_{2}\right) \oplus F\left(P_{3}\right) \oplus F\left(P_{4}\right)=0$.

For the boomerang on 6.5 rounds, we use a top trail on 3 rounds (from 0.5 to 3.5 ) with $2^{-40}$, and a bottom trail on 3.5 rounds (from 3.5 to 7 ), with $2^{-48}$ (see Table 5). The complexity of producing the boomerang quartet is $4 \cdot 2^{2 \cdot 40+2 \cdot 48+64}=2^{242}$ compression function calls. The probability of the first round in the top trail is $2^{-3}$, hence using message modification does not lower significantly the attack complexity. However, computing the amplified probabilities can improve the attack. Obviously, we cannot do this experimentally, as the probability of the boomerang is too low $-2^{-2 \cdot 40-2 \cdot 48}=2^{-176}$. Therefore, we cannot test for the whole 6.5 rounds, but we can do it for a reduced number of rounds. We tested for only half round at the end of the first trail (round 3 to round 3.5). We start with a pair of states with a difference specified by the top trail at round 3 and go half round forward to obtain a new pair of states. Then, to each element of the pair, we XOR the same difference (the one specified by the bottom trail at round 3.5), and produce a new pair states. Finally, we go backwards a half round, and check if the difference in the pair is at the one we have started with. Note that the half round can be split into four $G$ functions, and for each of them the amplified probabilities can be found independently. By doing so, we found that the amplified probability for this half round of the boomerang is $2^{-26}$ instead of twice $2^{-33}$, i.e. $2^{-2 \cdot 33}=2^{-66}$. Another low probability part of the boomerang is the top half round of the second trail - round 3.5 to round 4 holds with $2^{-41}$. In this part we can use message modification. We start at round 3.5 with four states that have pairwise differences $\Delta^{*}$ and $\nabla$. We go half round forward and obtain four states with pairwise differences as specified by the bottom trail at round 4 . To obtain such states we need $4 \cdot 2^{2 \cdot 41}=2^{84}$. 
Once we have this half round boomerang, we can freely change the message words that are not taken as inputs in this half round without altering the input and the output values of the half round. Hence, we have $2^{8 \cdot 32}=2^{256}$ degrees of freedom. From the middle states we can obtain the initial and final states (and the chaining values). Therefore, the total complexity of the boomerang on 6.5 rounds becomes $2^{84}+4 \cdot 2^{2 \cdot(3+1+3)+26+2 \cdot(6+1)+128}=2^{184}$ calls. Note that unlike as in the case of the boomerangs on 4 and 5 rounds, now the probability that the initial states are consistent to the Initialization is $2^{-128}$ because we use message modification in the middle rather than in the beginning. The bottom trail can easily be extended for additional half round (see Table 5) with probability $2^{-24}$. Therefore, the boomerang on 7 rounds requires around $2^{184+2 \cdot 24}=2^{232}$ compression function calls.

\section{Boomerang Attacks on the Keyed Permutation of BLAKE-32}

Further we present boomerang attacks on the keyed permutation of BLAKE-32, assuming that the key is unknown to the attacker. These attacks can be seen as distinguishers for the internal cipher of BLAKE-32. The cipher takes 512-bit plaintexts and 512-bit key, and after 10 rounds, outputs 512-bit ciphertext (we discard the Initializationand Finalization procedures).

Switching from the boomerangs for the compression function to the boomerangs for the keyed permutation has advantages and disadvantages for the attacker. On one hand, the attacker is not concern any more about the Initialization procedure, and he can use any trails for the boomerang. On the other hand, since the key is unknown, he cannot use message modification techniques to improve the probability of the boomerang.

The boomerangs on 4 and 5 rounds of the keyed permutation of BLAKE32 have the same probability as in the case of compression function: $2^{-4}$ for 4 rounds, and $2^{-20}$ for 5 rounds. For 6 rounds, we can use two high probability trails $\left(2^{-7}, 2^{-7}\right.$, see Table 6) , and therefore, the probability of the boomerang is $2^{-28}$. If we take into account the amplified probabilities, and fix the returning difference only in 128 bits (the words $v_{1}, v_{5}, v_{9}, v_{13}$ ) instead of in 512 bits, for the total complexity of the boomerang attack we get $2^{3}$ encryptions for 4 rounds, $2^{7.2}$ for 5 rounds, and $2^{11.75}$ for 6 rounds. These results were confirmed on a PC and a boomerang quartet for 6 rounds is presented in Table 8 .

The boomerangs for 7 and 8 rounds, are rather similar: for 7 rounds we use two trails on 3.5 rounds (the first from round 2 to round 5.5, and the second from round 5.5 to round 9 ), and for 8 rounds, we just extend these trails for additional half round (see Table 7 ). The complexity of the boomerangs is $4 \cdot 2^{2 \cdot 31+2 \cdot 52}=2^{168}$ for 7 rounds and $4 \cdot 2^{2 \cdot 73+2 \cdot 82}=2^{312}$ for 8 rounds. Again, as in the case of 6.5 round boomerang on the compression function, we can compute experimentally the lower bounds on the amplified probabilities, by testing only the probability 
of the first half round of the bottom trail. We get $2^{-48}$ instead of $2^{-2 \cdot 44}$. Also, we can fix the returning difference only in 256 bits, instead of 512 bits, and thus increase the probability in the first half round of the top trail by a factor of $2^{-6}$ for 7 rounds, and $2^{-30}$ for 8 rounds. Hence, the boomerang on 7 rounds requires at most $2^{122}$, and on 8 rounds at most $2^{242}$ encryptions.

\section{Conclusions}

In this paper we have shown how to apply the concept of boomerang distinguisher to compression functions, and presented such distinguishers for the compression function of BLAKE-32, as well as classical boomerang distinguishers for the keyed permutation of BLAKE-32. Our attacks work on up to $2 / 3$ of the total number of rounds of the compression function, and on up to $4 / 5$ (the attacks on up to $3 / 5$ have practical complexity) of the total number of rounds of the keyed permutation of BLAKE-32. The attacks can be equally well applied to the other versions of BLAKE. Our attacks do not contradict the security claims of BLAKE.

Interestingly, tweaking the message permutation in BLAKE can reduce the number of attacked rounds only by one. Therefore, either tweaks in the function $G$ or more advanced message expansion is required in order to significantly reduce the number of attacked rounds.

\section{References}

1. Aumasson, J.-P., Guo, J., Knellwolf, S., Matusiewicz, K., Meier, W.: Differential and invertibility properties of BLAKE. In: Hong, S., Iwata, T. (eds.) FSE 2010. LNCS, vol. 6147, pp. 318-332. Springer, Heidelberg (2010)

2. Aumasson, J.-P., Henzen, L., Meier, W., Phan, R.C.-W.: SHA-3 proposal BLAKE. Submission to NIST (2008)

3. Ji, L., Liangyu, X.: Attacks on round-reduced BLAKE. Cryptology ePrint Archive, Report 2009/238 (2009), http://eprint.iacr.org/2009/238.pdf

4. Joux, A., Peyrin, T.: Hash functions and the (amplified) boomerang attack. In: Menezes, A. (ed.) CRYPTO 2007. LNCS, vol. 4622, pp. 244-263. Springer, Heidelberg (2007)

5. Kelsey, J., Kohno, T., Schneier, B.: Amplified boomerang attacks against reducedround MARS and serpent. In: Schneier, B. (ed.) FSE 2000. LNCS, vol. 1978, pp. 75-93. Springer, Heidelberg (2001)

6. National Institute of Standards and Technology. Cryptographic hash algorithm competition, http://csrc.nist.gov/groups/ST/hash/sha-3/index.html

7. Su, B., Wu, W., Wu, S., Dong, L.: Near-collisions on the reduced-round compression functions of Skein and BLAKE. Cryptology ePrint Archive, Report 2010/355 (2010), http://eprint.iacr.org/2010/355.pdf

8. Wagner, D.: The boomerang attack. In: Knudsen, L.R. (ed.) FSE 1999. LNCS, vol. 1636, pp. 156-170. Springer, Heidelberg (1999) 


\section{A Differential Trails for the Boomerangs}

Table 2. Differential trails used in the Boomerang Attack on 4 rounds of BLAKE-32. On the left is the top trail, while on the right is the bottom trail of the boomerang. $\Delta M$ is the message difference, while $\Delta V_{i}$ are the differences in the state. In the left trail (top trail), $\Delta V_{0}$ is the starting difference of the trail, i.e. $\Delta V_{0}=\Delta$, and $\Delta V_{2}$ is the ending difference, i.e. $\Delta V_{2}=\Delta^{*}$. In the right trail (bottom trail), $\Delta V_{2}$ is the starting difference of the trail, i.e. $\Delta V_{2}=\nabla$, and $\Delta V_{4}$ is the ending difference, i.e. $\Delta V_{4}=\nabla^{*}$. The numbers $0,1,2$, and $2,3,4$, indicate the rounds covered by the boomerang - the top trail starts at round 0 and ends after round 1, while the bottom trail starts at round 2 and ends after round 3.

\begin{tabular}{|c|c|c|c|}
\hline & $\Delta m$ & & $\Delta m$ \\
\hline & 00000000000000008000000000000000 & & 00000000000000000000000000000000 \\
\hline & 00000000000000000000000000000000 & & 00000000000000000000000000000000 \\
\hline & 00000000000000000000000000000000 & & 80000000000000000000000000000000 \\
\hline & 00000000000000000000000000000000 & & 00000000000000000000000000000000 \\
\hline$R$. & $\overline{\Delta V_{i}}$ & $R$. & $\overline{\Delta \Delta V_{i}}$ \\
\hline 0 & 00000000800000000000000000000000 & 2 & 80000000000000000000000000000000 \\
\hline & 00000000000000000000000000000000 & & 00000000000000000000000000000000 \\
\hline & 00000000000000000000000000000000 & & 00000000000000000000000000000000 \\
\hline & 00000000000000000000000000000000 & & 80000000000000000000000000000000 \\
\hline & 1 & & 1 \\
\hline 1 & 0000000000000000000000000000 & 3 & 000000000000000000000000000 \\
\hline & 00000000000000000000000000000000 & & 000000000000000000000000000 \\
\hline & 000000000000000000000000000 & & 00000000000000000000000000000000 \\
\hline & 00000000000000000000000000000000 & & 00000000000000000000000000000000 \\
\hline & $2^{-1}$ & & $2^{-1}$ \\
\hline 2 & 00000000800000000000000000000000 & 4 & 00000000000000000000000080000000 \\
\hline & 00000010000000 & & 000100000000000000000000000 \\
\hline & 000000000000000000000000008 & & 00000000008000000000000000000000 \\
\hline & 00800000000000000000000000000000 & & 00000000000000000080000000000000 \\
\hline
\end{tabular}


Table 3. Differential trails used in the Boomerang Attack on 5 rounds of BLAKE-32

\begin{tabular}{|c|c|c|c|}
\hline & $\Delta m$ & & $\Delta m$ \\
\hline & $\begin{array}{|llll|}00000000 & 00000000 & 40000000 & 00000000 \\
00000000 & 00000000 & 00000000 & 00000000 \\
00000000 & 00000000 & 00000000 & 00000000 \\
00000000 & 00000000 & 00000000 & 00000000\end{array}$ & & \begin{tabular}{|lllll}
00000000 & 00000000 & 00000000 & 00000000 \\
00000000 & 00000000 & 00000000 & 00000000 \\
00000000 & 00000000 & 00000000 & 00000000 \\
00000000 & 80000000 & 00000000 & 00000000 \\
\end{tabular} \\
\hline$R$. & $\overline{\Delta V_{i}}$ & $R$. & $\overline{\overline{\Delta V_{i}}}$ \\
\hline \begin{tabular}{|l|}
0 \\
\end{tabular} & $\left|\begin{array}{llll|}00000000 & 40000000 & 00000000 & 00000000 \\
00000000 & 00000000 & 00000000 & 00000000 \\
00000000 & 00000000 & 00000000 & 00000000 \\
00000000 & 00000000 & 00000000 & 00000000\end{array}\right|$ & 2 & $\begin{array}{|llll|}00000800 & 80008000 & 80000000 & 80000000 \\
80000800 & 80008000 & 00000000 & 00000000 \\
80000000 & 80808080 & 80000000 & 00000000 \\
80000000 & 00800080 & 80008000 & 80000000\end{array}$ \\
\hline & $2^{-1}$ & & $2^{-6}$ \\
\hline \begin{tabular}{|l|}
1 \\
\end{tabular} & $\begin{array}{|llll|}00000000 & 00000000 & 00000000 & 00000000 \\
00000000 & 00000000 & 00000000 & 00000000 \\
00000000 & 00000000 & 00000000 & 00000000 \\
00000000 & 00000000 & 00000000 & 00000000\end{array}$ & 3 & \begin{tabular}{|lllll}
00000000 & 00000000 & 80000000 & 00000000 \\
00000000 & 00000000 & 00000000 & 00000000 \\
00000000 & 00000000 & 00000000 & 00000000 \\
00000000 & 00000000 & 00000000 & 00000000
\end{tabular} \\
\hline & $2^{-}$ & & 1 \\
\hline 2 & $\begin{array}{|llll|}00000000 & 40000000 & 00000000 & 00000000 \\
00000000 & 00000000 & 00008000 & 00000000 \\
00000000 & 00000000 & 00000000 & 00400000 \\
00400000 & 00000000 & 00000000 & 00000000\end{array}$ & 4 & \begin{tabular}{|lllll}
00000000 & 00000000 & 00000000 & 00000000 \\
00000000 & 00000000 & 00000000 & 00000000 \\
00000000 & 00000000 & 00000000 & 00000000 \\
00000000 & 00000000 & 00000000 & 00000000
\end{tabular} \\
\hline & & & 2 \\
\hline & & 5 & \begin{tabular}{|lllll}
00000000 & 00000000 & 00000000 & 80000000 \\
00010000 & 00000000 & 00000000 & 00000000 \\
00000000 & 00800000 & 00000000 & 00000000 \\
00000000 & 00000000 & 00800000 & 00000000
\end{tabular} \\
\hline
\end{tabular}

Table 4. Differential trails used in the Boomerang Attack on 6 rounds of CF of BLAKE-32

\begin{tabular}{|c|c|c|c|}
\hline & $\Delta m$ & & $\Delta m$ \\
\hline & 0800080000000000000000000 & & 000000000000000000000 \\
\hline & 00000 & & 00000 \\
\hline & 00000000000000000 & & 000000 \\
\hline & 000000000000000000000 & & 0000000000000000800 \\
\hline $\bar{R}$. & $\overline{\overline{\Delta V_{i}}}$ & $\overline{R .}$ & $\overline{\overline{\Delta V_{i}}}$ \\
\hline 4 & 088008000000000008 & 7 & $\overline{000000}$ \\
\hline & 080000000000000000000 & & 000000 \\
\hline & 10800080000000000080008000 & & 8080000000000 \\
\hline & 00000000000000000000 & & 080000080000000 \\
\hline & & & $2^{-6}$ \\
\hline 5 & $\overline{000}$ & 8 & 0000 \\
\hline & 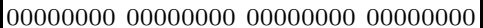 & & 000000000000 \\
\hline & 0000000000000000000000000 & & 00000000000 \\
\hline & 000000000000000000000000 & & 0000000000000000000 \\
\hline & $2^{-2}$ & & 1 \\
\hline 6 & 00000000000 & 9 & 00000000000 \\
\hline & 000000000000 & & 00000000000 \\
\hline & 0000000000000000000 & & 0000000 \\
\hline & 000000000000000000000 & & 00000000000000000000000000000000 \\
\hline & $2^{-11}$ & & $2^{-1}$ \\
\hline 7 & 0000000 & & 0000000 \\
\hline & 10 & & 000000 \\
\hline & & & 00000 \\
\hline & 000000000000000000 & & 00800000000 \\
\hline
\end{tabular}


Table 5. Differential trails used in the Boomerang Attack on 6.5 and 7 rounds of CF of BLAKE-32

\begin{tabular}{|c|c|c|c|}
\hline & $\Delta m$ & & $\Delta m$ \\
\hline & 00000000000000000000000000000000 & & 00000000000000008000000000000000 \\
\hline & 80008000000000000000000000000000 & & 00000000000000000000000000000000 \\
\hline & 00000000000000000000000000000000 & & 00000000000000000000000000000000 \\
\hline & 00000000000000000000000000000000 & & 00000000000000000000000000000000 \\
\hline \begin{tabular}{|l|}
$R$. \\
\end{tabular} & $\overline{\Delta \Delta V_{i}}$ & $R$. & $\overline{\Delta \Delta V_{i}}$ \\
\hline 0.5 & 00000000800080000000000000000000 & 3.5 & $00800880 c 80888488044004400008000$ \\
\hline & 00000000000000000000000000000000 & & $80000000 \quad 80800880 \quad 488 c 0888 \quad 80040804$ \\
\hline & 00000000000000000000000080008000 & & 00000800000080808080808000000000 \\
\hline & 00000000000000000000000000000000 & & $80048040 \quad 084088400080000080000000$ \\
\hline & $2^{-3}$ & & \\
\hline 1 & 00000000800080000000000000000000 & 4 & 80000000000000008000080080008000 \\
\hline & 00000000000000000000000000000000 & & 00000000000000008000080080008000 \\
\hline & 00000000000000000000000000000000 & & 80000000000000008000000080808080 \\
\hline & 00000000000000000000000000000000 & & 80008000000000008000000000800080 \\
\hline & $2^{-1}$ & & $2^{-6}$ \\
\hline 2 & 00000000000000000000000000000000 & 5 & 80000000000000000000000000000000 \\
\hline & 00000000000000000000000000000000 & & 00000000000000000000000000000000 \\
\hline & 00000000000000000000000000000000 & & 00000000000000000000000000000000 \\
\hline & 00000000000000000000000000000000 & & 00000000000000000000000000000000 \\
\hline & $2^{-3}$ & & 1 \\
\hline 3 & 00000000000000000000000080008000 & 6 & 00000000000000000000000000000000 \\
\hline & 00010001000000000000000000000000 & & 00000000000000000000000000000000 \\
\hline & 00000000008000800000000000000000 & & 00000000000000000000000000000000 \\
\hline & 00000000000000000080008000000000 & & 00000000000000000000000000000000 \\
\hline & $2^{-33}$ & & $2^{-1}$ \\
\hline 3.5 & \begin{tabular}{|cccccc}
00010001 & 08000800 & 08000800 & 80088008
\end{tabular} & 7 & 00000000000000008000000000000000 \\
\hline & 02000200101110111111111111101110 & & 00000000000000000000000000010000 \\
\hline & 00010001008800888088808888008800 & & 00800000000000000000000000000000 \\
\hline & 00000000000800088008800808000800 & & 00000000008000000000000000000000 \\
\hline & & & $2^{-24}$ \\
\hline & & & $\begin{array}{lllll}00000800 & 08000000 & 80000008 & 00110010 \\
10010010 & 01101001 & 10110101 & 22222022 \\
00800008 & 80080080 & 08808080 & 11001101 \\
00000008 & 80080000 & 08800080 & 11001100\end{array}$ \\
\hline
\end{tabular}


Table 6. Differential trails used in the Boomerang Attack on 6 rounds of KP of BLAKE-32

\begin{tabular}{|c|c|c|c|}
\hline & $\Delta m$ & & $\Delta m$ \\
\hline & $\begin{array}{llll}000000 & 00000000 & 00000000 & 000 c \\
000000 & 00000000 & 00000000 & 000 c \\
000000 & 00000000 & 00000000 & 000 c \\
000000 & 00000000 & 00000000 & 000(\end{array}$ & & $\begin{array}{llll}0000000 & 00000000 & 00000000 & 00 \\
0000000 & 80000000 & 00000000 & 00 \\
0000000 & 00000000 & 00000000 & 00 \\
0000000 & 00000000 & 00000000 & 00\end{array}$ \\
\hline$\pi$. & 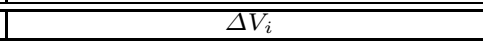 & $R$. & $\overline{\Delta \Delta V_{i}}$ \\
\hline \begin{tabular}{|l|}
0 \\
\end{tabular} & $\begin{array}{|llll|}80008000 & 80000000 & 80000000 & 000 \\
80008000 & 00000000 & 00000000 & 800 \\
80808080 & 80000000 & 00000000 & 800 \\
00800080 & 80008000 & 00000000 & 800 \\
\end{array}$ & 3 & \begin{tabular}{|llll}
80008000 & 00000000 & 00000000 & 000 \\
80008000 & 00000000 & 00000000 & 800 \\
80808080 & 00000000 & 00000000 & 800 \\
00800080 & 00000000 & 00000000 & 800 \\
\end{tabular} \\
\hline & $2^{-6}$ & & $2^{-6}$ \\
\hline 1 & \begin{tabular}{|llll}
00000000 & 80000000 & 00000000 & 000 \\
00000000 & 00000000 & 00000000 & 000 \\
00000000 & 00000000 & 00000000 & 000 \\
00000000 & 00000000 & 00000000 & 000
\end{tabular} & 4 & \begin{tabular}{|lllll}
00000000 & 80000000 & 00000000 & 00000000 \\
00000000 & 00000000 & 00000000 & 00000000 \\
00000000 & 00000000 & 00000000 & 00000000 \\
00000000 & 00000000 & 00000000 & 00000000
\end{tabular} \\
\hline & 1 & & 1 \\
\hline 2 & $\begin{array}{lll}00000000 & 00000000 & 0000 \\
00000000 & 00000000 & 0000 \\
00000000 & 00000000 & 0000 \\
00000000 & 00000000 & 0000\end{array}$ & 5 & $\begin{array}{lll}00000000 & 00000000 & 0000 \\
00000000 & 00000000 & 0000 \\
00000000 & 00000000 & 0000 \\
00000000 & 00000000 & 0000\end{array}$ \\
\hline & $2^{-}$ & & 2 \\
\hline & $\begin{array}{lllll}00000000 & 00000000 & 00000000 & 80000000 \\
00010000 & 00000000 & 00000000 & 00000000 \\
00000000 & 00800000 & 00000000 & 00000000 \\
00000000 & 00000000 & 00800000 & 00000000\end{array}$ & 6 & \begin{tabular}{|lllll}
00000000 & 80000000 & 00000000 & 00000000 \\
00000000 & 00000000 & 00001000 & 00000000 \\
00000000 & 00000000 & 00000000 & 00800000 \\
00800000 & 00000000 & 00000000 & 00000000
\end{tabular} \\
\hline
\end{tabular}


Table 7. Differential trails used in the Boomerang Attack on 7 and 8 rounds of KP of BLAKE-32

\begin{tabular}{|c|c|c|c|}
\hline & $\Delta m$ & & $\Delta m$ \\
\hline & 00000000000000000000000000000000 & & 00000000000000000000000000000000 \\
\hline & 00000000000000000000000000000000 & & 00000000000000000000000080000000 \\
\hline & 00000000000000000000000000000000 & & 00000000000000000000000000000000 \\
\hline & 00000000800000000000000000000000 & & 00000000000000000000000000000000 \\
\hline$R$. & $\overline{\Delta V_{i}}$ & $R$. & $\Delta V_{i}$ \\
\hline 1.5 & 80440044000080008080088048088848 & 5.5 & $8080800080888080 \quad c 80 c 8008 \quad 80440044$ \\
\hline & $488 c 0888000408048000000080800880$ & & $800408048080000080888000 c 8880088$ \\
\hline & 80808080800000000000088000008080 & & $80000000 \quad 000008000080808080000080$ \\
\hline & $00800000800000000004004088 c 00840$ & & 00800000000480000840884080800000 \\
\hline & & & \\
\hline 2 & 00000800800080008000000080000000 & 6 & 00008000800000000000000080000800 \\
\hline & 80000800800080000000000000000000 & & 80008000000000000000000000000800 \\
\hline & 80000000808080808000000000000000 & & 00808080800000000000000000000080 \\
\hline & 80000000008000808000800080000000 & & 80808080800080000000000080800000 \\
\hline & $2^{-6}$ & & 2 \\
\hline 3 & 00000000000000008000000000000000 & 7 & 00000000800000000000000000000000 \\
\hline & 00000000000000000000000000000000 & & 00000000000000000000000000000000 \\
\hline & 00000000000000000000000000000000 & & 00000000000000000000000000000000 \\
\hline & 00000000000000000000000000000000 & & 00000000000000000000000000000000 \\
\hline & 1 & & 1 \\
\hline 4 & 00000000000000000000000000000000 & 8 & 00000000000000000000000000000000 \\
\hline & 00000000000000000000000000000000 & & 00000000000000000000000000000000 \\
\hline & 00000000000000000000000000000000 & & 00000000000000000000000000000000 \\
\hline & 00000000000000000000000000000000 & & 00000000000000000000000000000000 \\
\hline & $2^{-1}$ & & $2^{-1}$ \\
\hline 5 & 00000000000000000000000080000000 & 9 & 00000000800000000000000000000000 \\
\hline & 00010000000000000000000000000000 & & 00000000000000000001000000000000 \\
\hline & 00000000008000000000000000000000 & & 00000000000000000000000000800000 \\
\hline & 00000000000000000080000000000000 & & 00800000000000000000000000000000 \\
\hline & $2^{-24}$ & & $2^{-30}$ \\
\hline 5.5 & 00110010000008000800000080000008 & 9.5 & 08000000800000088011001800000800 \\
\hline & 22222022100100100110100110110101 & & 01101001101101013233212310010010 \\
\hline & 11001101008000088008008008808080 & & 80080080088080801980918100800008 \\
\hline & $11001100 \quad 000000088008000008800080$ & & $80080000 \quad 088000801980118000000008$ \\
\hline
\end{tabular}




\section{B Examples of Boomerang quartets}

Table 8. Example of a boomerang quartet for 6 round-reduced keyed permutation of BLAKE-32

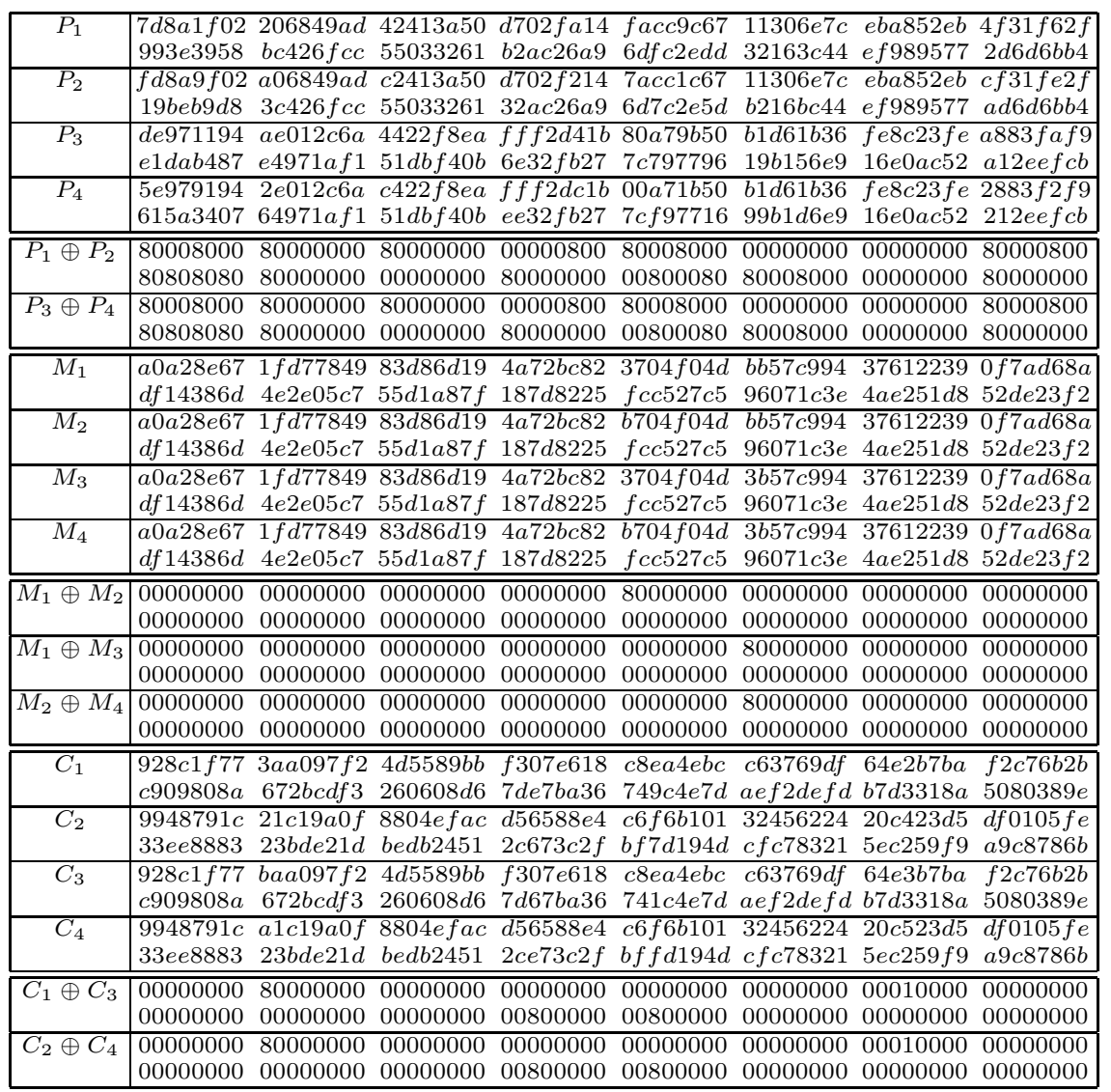


Table 9. Example of a boomerang quartet for 6 round-reduced compression function of BLAKE-32. Note that the initial states $P_{1}, P_{2}$ are consistent with the Initialization.

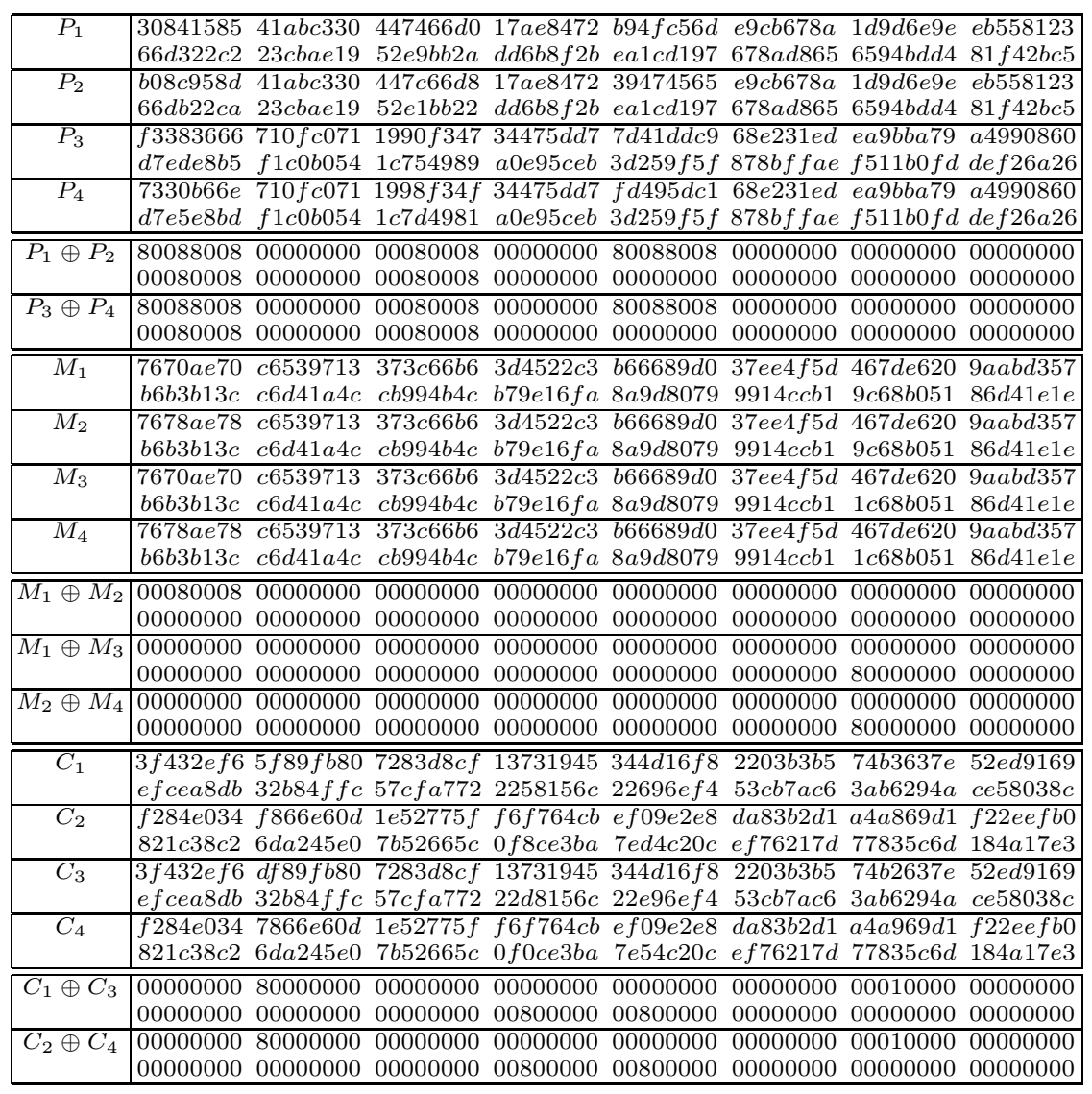

